\title{
Neutron capture measurements on TI-isotopes at DANCE
}

\author{
A. Couture ${ }^{1, \mathrm{a}}$, R. Reifarth ${ }^{1, \mathrm{~b}}$, J.D. Baker ${ }^{2}$, T.A. Bredeweg ${ }^{1}$, R.C. Haight ${ }^{1}$, M. Jandel ${ }^{1}$, A.F. Mertz ${ }^{1, \mathrm{c}}$, J.M. O’Donnell ${ }^{1}$, \\ R.S. Rundberg ${ }^{1}$, J.L. Ullmann ${ }^{1}$, D.J. Viera ${ }^{1}$, and J.M. Wouters ${ }^{1}$ \\ 1 Los Alamos National Laboratory, Los Alamos, NM 87545, USA \\ 2 Idaho National Laboratory, Idaho Falls, ID 83415, USA
}

\begin{abstract}
The thallium isotopes play an important role in the $s$-process nucleosynthesis at the $s$-process endpoint. Furthermore, ${ }^{204} \mathrm{Tl}$ is one of few branch point isotopes in the endpoint region. The understanding of branch point isotopes provides modeling constraints on the temperatures and neutron densities during which the process takes place. The production of $s$-only ${ }^{204} \mathrm{~Pb}$ is controlled almost entirely by ${ }^{204} \mathrm{Tl}$. Measurements of the capture crosssections of the stable $\mathrm{Tl}$ isotopes have recently been made using the DANCE $4 \pi$ array at LANSCE. This provides needed resonance information in the region as well as preparing the way for measurements of as yet unmeasured capture cross-section of the unstable ${ }^{204} \mathrm{Tl}$.
\end{abstract}

\section{Introduction}

About $50 \%$ of the element abundances beyond iron are produced via slow neutron capture nucleosynthesis ( $s$ process). Starting at iron-peak seed, the $s$-process mass flow follows the neutron-rich side of the valley of stability. If different reaction rates for the same isotope are comparable, the $s$-process path branches and the branching ratio reflects the physical conditions in the interior of the star. Such nuclei are most interesting, because they provide the tools to effectively constrain modern models of the stars where the nucleosynthesis occurs. As soon as the $\beta^{-}$decay is faster than the typically competing neutron capture, no branching will take place. Thus experimental neutron capture data for the $s$ process are only needed if the respective neutron capture time under stellar conditions is similar to or shorter than the $\beta^{-}$decay time; this includes all stable isotopes. Depending on the actual neutron density during the $s$ process, the "line of interest" is closer to or farther away from the valley of stability. Figure 1 shows a summary of the neutron capture and $\beta^{-}$decay times for radioactive isotopes on the neutron rich side of the valley of stability, under the condition that the classical neutron capture occurs faster than the terrestrial $\beta^{-}$decay. The vast majority of isotopes where an experimental neutron capture cross section is desirable have $\beta^{-}$half-lives of at least hundreds of days. Such isotopes can be investigated with the DANCE array.

Since the neutron densities during the $s$ process are not high enough the overcome the $\alpha$-unstable isotopes beyond ${ }^{209} \mathrm{Bi}$, the $\mathrm{Tl}-\mathrm{Pb}-\mathrm{Bi}$ group marks the termination region of the s-process (fig. 2). The production of the $s$-only ${ }^{204} \mathrm{~Pb}$ is mostly determined by the branching at ${ }^{204} \mathrm{Tl}$. Another interesting branching occurs at ${ }^{205} \mathrm{~Pb}$, especially since ${ }^{205} \mathrm{Tl}$, which is stable under laboratory conditions, becomes unstable under stellar conditions $[2,3]$. The stellar site for the production

\footnotetext{
${ }^{a}$ e-mail: acouture@lanl.gov

${ }^{b}$ Presenting author, e-mail: r.reifarth@gsi.de, Present address: Gesellschaft für Schwerionenforschung mbH, Darmstadt, 64291, Germany

${ }^{c}$ Present address: Yale University, Department of Physics, New Haven, CT 06520, USA
}

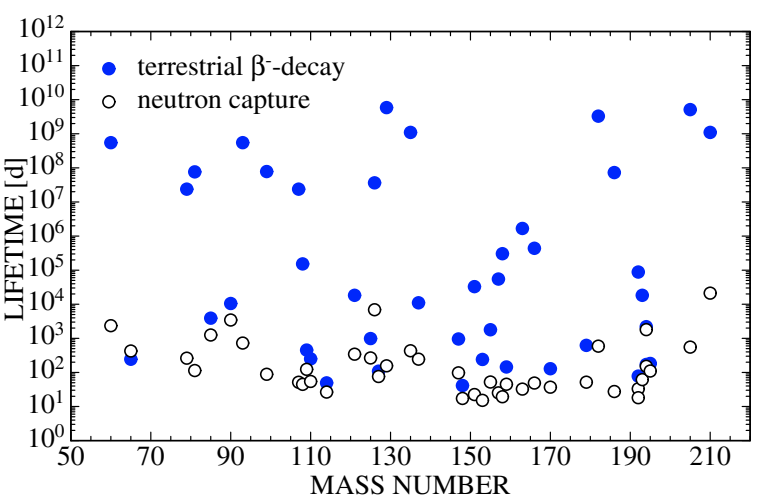

Fig. 1. Neutron capture life times (open circles) and terrestrial $\beta^{-}$life times (filled circles) for unstable isotopes on the classical $s$-process path as a function of mass number. Shown are only isotopes where the neutron capture is faster than the stellar $\beta^{-}$decay for a neutron density of $5 \cdot 10^{8} \mathrm{~cm}^{-3}$ at a temperature of $30 \mathrm{keV}$. The neutron capture cross sections are mostly taken from [1] and the stellar decay rates from [2].

of these isotopes are metal-poor Asymptotic Giant Branch (AGB) stars $[4,5]$. Both radioactive isotopes are very difficult to produce under terrestrial conditions. Irradiating Bi samples in close proximity of a spallation source and chemically separating the freshly produced material from the bismuth would be a very good solution, especially for ${ }^{204} \mathrm{Tl}$. Further enrichment by mass separation is not necessary, since ${ }^{204} \mathrm{Tl}$ has the highest neutron capture Q-value of all relevant $\mathrm{Tl}$ isotopes. The neutron capture measurements on ${ }^{203,205} \mathrm{Tl}$ described in this article offer a significant improvement in the neutron capture data on the stable isotopes of thallium, which is important since the present data are limited in resolution and the resolved resonance region reaches above $10 \mathrm{keV}$, particularly for ${ }^{205} \mathrm{Tl}$. In addition, the present measurements are in preparation for a ${ }^{204} \mathrm{Tl}(\mathrm{n}, \gamma)$ measurement at a later stage.

\section{The DANCE array}

The Detector for Advanced Neutron Capture Experiments (DANCE) is designed as a high efficiency, highly segmented 


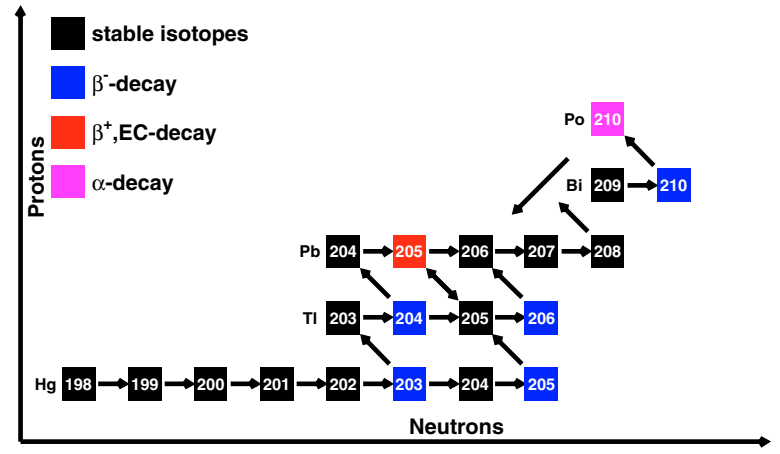

Fig. 2. The s-process path at its termination region between mercury and polonium.

$4 \pi \mathrm{BaF}_{2}$ detector for calorimetrically detecting $\gamma$-rays following a neutron capture. DANCE is located on the $20 \mathrm{~m}$ neutron flight path 14 (FP14) at the Manuel Lujan Jr. Neutron Scattering Center at the Los Alamos Neutron Science Center (LANSCE) [6]. The initial design work is described in [7]. For practical reasons the detector modules do not cover the entire solid angle. The design of the detector is such that a full $4 \pi$ array would consist of 162 crystals of four different shapes, each shape covering the same solid angle [8]. Two of the 162 crystals are left out in order to leave space for the neutron beam pipe. Depending on the experiment, one crystal can be replaced by a sample changer mechanism, which makes it possible to exchange up to 3 samples without closing the beam shutter and breaking the vacuum of the beam pipe. Thus the full array is designed to host 159 or 160 out of 162 possible $\mathrm{BaF}_{2}$ crystals. The dimensions of the bare crystals are designed to form a $\mathrm{BaF}_{2}$ shell with an inner radius of $17 \mathrm{~cm}$ and a thickness of $15 \mathrm{~cm}$. Thanks to the fairly low repetition rate of $20 \mathrm{~Hz}$, measurements can be carried out over the whole energy range from $10 \mathrm{meV}$ to $500 \mathrm{keV}$. This combination of a strong neutron source and a high efficiency $\gamma$-ray detector allows to measure (n, $\gamma$ ) cross section of radioactive isotopes down to a few hundred days half-life. Further details on the overall performance of the array can be found in $[9,10]$.

\section{Samples}

Altogether we used four thallium samples. Since the cross section changes over several orders of magnitude over the investigated energy range, we used two ${ }^{203} \mathrm{Tl}$ samples with $7.5 \mathrm{mg}$ and $51 \mathrm{mg}$ and two ${ }^{205} \mathrm{Tl}$ samples with $9.5 \mathrm{mg}$ and $53 \mathrm{mg}$. The smaller samples were dried out of a solution onto a thin mylar foil, while the larger ones were small pieces of thallium mounted on a thin mylar foil. Therefore the amount of material per area was much lower for the small samples, hence they were much better suited for analyzing the strong resonances. All of the samples were sealed with VYNS to prevent the thallium from migrating or flaking. Table 1 contains the details for each of the samples.

\section{Experiment}

The data analysis is still in progress. Therefore only preliminary data will be presented here. In total, about 30 days of beam time were spent on ${ }^{203,205} \mathrm{Tl},{ }^{197} \mathrm{Au}$, and background runs.
Table 1. Masses and isotopic composition of the four samples used during the experiment.

\begin{tabular}{cccc}
\hline $\begin{array}{c}\text { sample } \\
\text { nbr. }\end{array}$ & $\begin{array}{c}\text { mass } \\
(\mathrm{mg})\end{array}$ & $\begin{array}{c}{ }^{203} \mathrm{Tl} \\
(\%)\end{array}$ & $\begin{array}{c}{ }^{205} \mathrm{Tl} \\
(\%)\end{array}$ \\
\hline 1 & 51 & $97.15 \pm 0.04$ & 2.85 \\
2 & 7.5 & $97.15 \pm 0.04$ & 2.85 \\
3 & 53 & 0.26 & $99.74 \pm 0.01$ \\
4 & 9.5 & 0.26 & $99.74 \pm 0.01$ \\
\hline
\end{tabular}

\section{Preliminary results}

\section{$5.1^{203} \mathrm{TI}$}

The experimental information on the ${ }^{203} \mathrm{Tl}(\mathrm{n}, \gamma)$ reaction above $1 \mathrm{keV}$ is sparse (see fig. 3). Based on the measurements and evaluation by Macklin and Winters [11], the recommended value for the Maxwellian averaged cross section (MACS) at $30 \mathrm{keV}$ by Bao et al. is $124 \pm 8 \mathrm{mb}$ [1].

Figure 4 shows the response of the DANCE array during the experiment for low neutron energies. Shown is the total

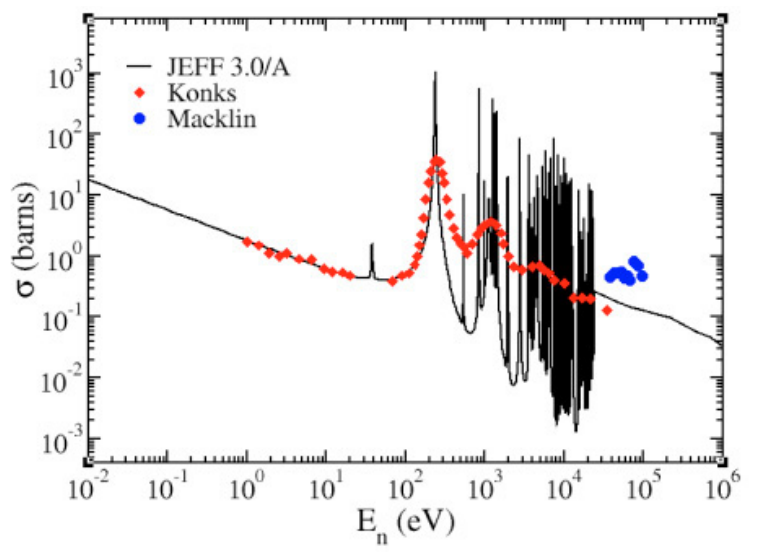

Fig. 3. Experimental $[11,12]$ data as reported at EXFOR and evaluated [13] data for the ${ }^{203} \mathrm{Tl}(\mathrm{n}, \gamma)$ reaction in the higher energy region before this measurement. Additionally resonance parameters between 3 and $13 \mathrm{keV}$ are reported [11].

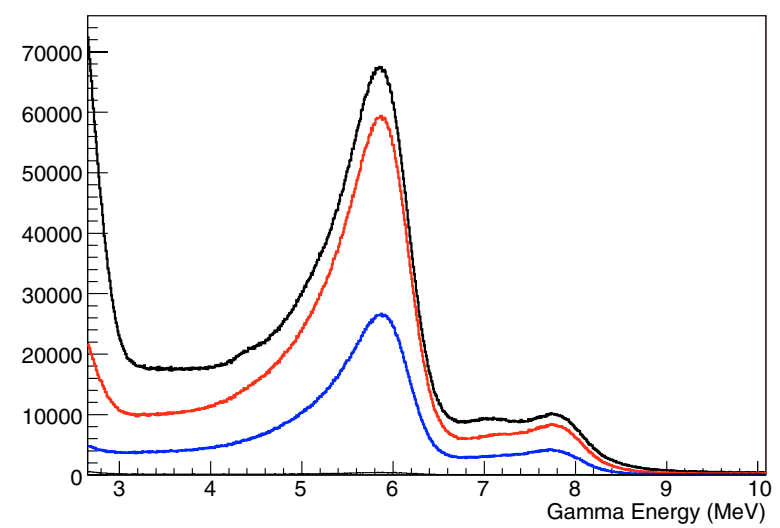

Fig. 4. Total energy deposited in the DANCE array for the ${ }^{203} \mathrm{Tl}$ sample for low neutron energies between $10 \mathrm{meV}$ and $1 \mathrm{keV}$. The different curves correspond to different multiplicities: Multiplicity 1-3 from top to bottom. 


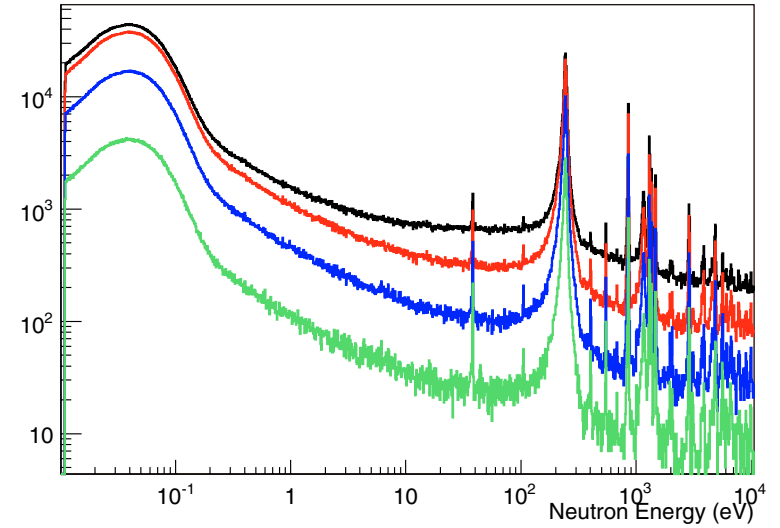

Fig. 5. Time-of-flight spectrum for ${ }^{203} \mathrm{Tl}$ with an cut on the total deposited energy between 5 and $6.5 \mathrm{meV}$. The different curves correspond to multiplicities 1 (top) to 4 (bottom).

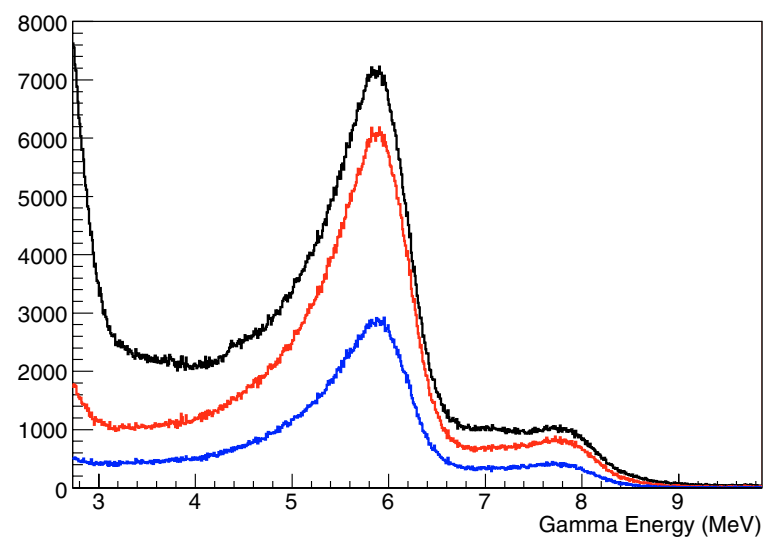

Fig. 6. Total energy deposited in the DANCE array for the ${ }^{203} \mathrm{Tl}$ sample for high neutron energies between $30 \mathrm{eV}$ and $200 \mathrm{keV}$. The different curves correspond to different multiplicities: Multiplicity 1-3 from top to bottom

energy deposited in the detector as a function of cluster multiplicity, which is very close to the multiplicity of the emitted $\gamma$-rays [7,9]. The Q-value of the reaction is $6.65 \mathrm{meV}$, resulting in the pronounced peaks between 5 and $6.5 \mathrm{meV}$. A gate on these peaks improves the signal-to-background ratio. The events above $7 \mathrm{meV}$ result from captures of scattered neutrons in the $\mathrm{BaF}_{2}$ crystals.

Figure 5 shows results of a total-energy cut on time-offlight (TOF) spectrum of different multiplicities. The signalto-background improves with increasing multiplicity since most background components tend to yield low multiplicities [9]. The trade-off typically is reduced statistics.

Figure 6 shows a similar spectrum as figure 4 , but for higher neutron energies. The capture-to-scatter ratio is typically decreasing with increasing neutron energy, which makes neutron capture measurements increasingly difficult. This effect is not very pronounced for ${ }^{203} \mathrm{Tl}(\mathrm{n}, \gamma)$, but can still be observed.

Figure 7 shows a TOF spectrum for the events shown in figure 6 with an additional total-energy cut between 5 and $6.5 \mathrm{meV}$. Data have been successfully taken up to $200 \mathrm{keV}$ neutron energy.

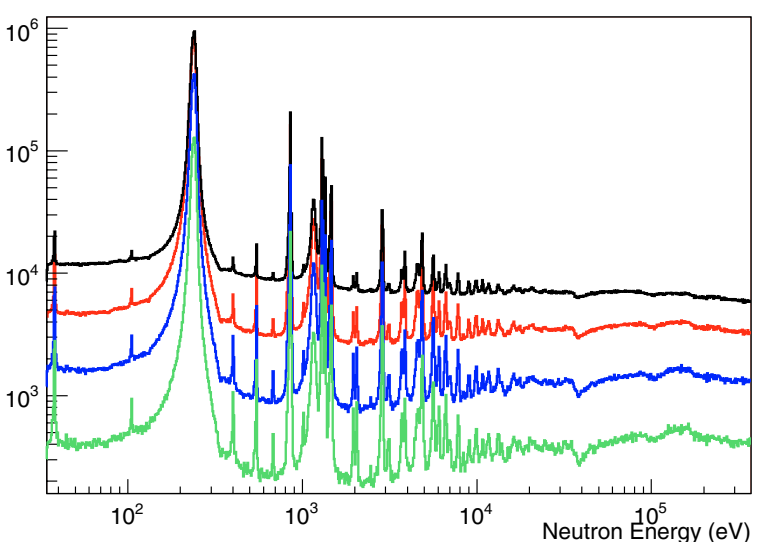

Fig. 7. Time-of-flight spectrum for ${ }^{203} \mathrm{Tl}$ and multiplicities 1 to 4 (top to bottom).

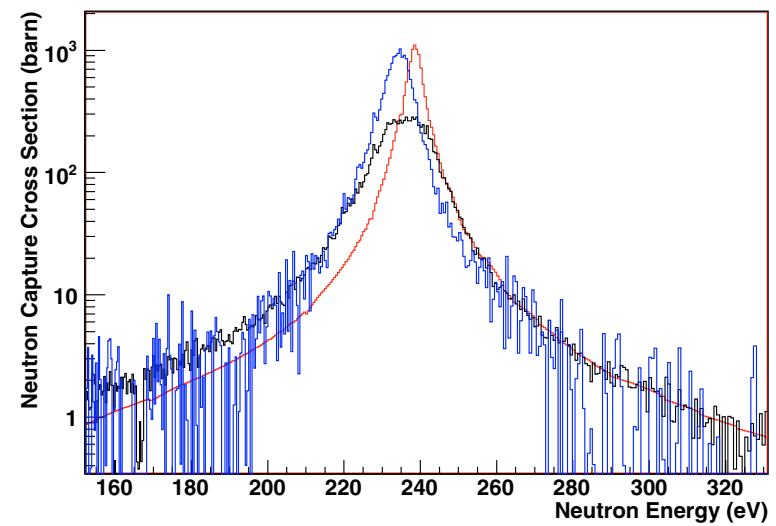

Fig. 8. Neutron capture cross section for ${ }^{203} \mathrm{Tl}$ derived from DANCE (black, blue) (multiplicity cut from 1 to 4 and total-energy cut from 5 to $6.5 \mathrm{meV}$ ) and a comparison with evaluated data (red) [13] for the biggest resonance in the ${ }^{203} \mathrm{Tl}(\mathrm{n}, \gamma)$ cross section (see fig. 6). The data for the black curve were taken with sample 1 (see table 1) and are not corrected for self-absorption, while the data for the blue curve were taken with the much thinner sample 2 .

Figure 8 shows the conversion from TOF spectrum to cross section in the neutron energy region around the biggest resonance of the ${ }^{203} \mathrm{Tl}(\mathrm{n}, \gamma)$ reaction. The plot illustrates the advantage of using samples of different thickness. Significant self-absorption corrections would be necessary for sample 1, while sample 2 reproduces the shape of the resonance as suggested by the JEFF-3.0/A evaluation [13]. The final data will be derived from the smaller sample 2 for the biggest resonances and from the bigger sample 1 elsewhere (see also table 1).

\section{$5.2^{205} \mathrm{TI}$}

Similar to the situation for ${ }^{203} \mathrm{Tl}$, the experimental information on the ${ }^{205} \mathrm{Tl}(\mathrm{n}, \gamma)$ reaction above $1 \mathrm{keV}$ is sparse (see fig. 9). Based on the measurements and evaluation by Macklin and Winters [11], the recommended value for the Maxwellian averaged cross section (MACS) at $30 \mathrm{keV}$ by Bao et al. is $54 \pm 4 \mathrm{mb}$ [1] 


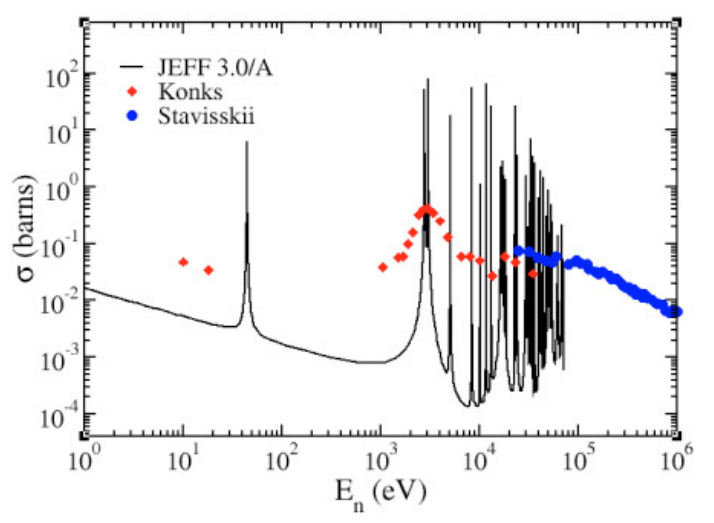

Fig. 9. Experimental $[12,14]$ data as reported at EXFOR and evaluated [13] data for the ${ }^{205} \mathrm{Tl}(\mathrm{n}, \gamma)$ reaction before this measurement.

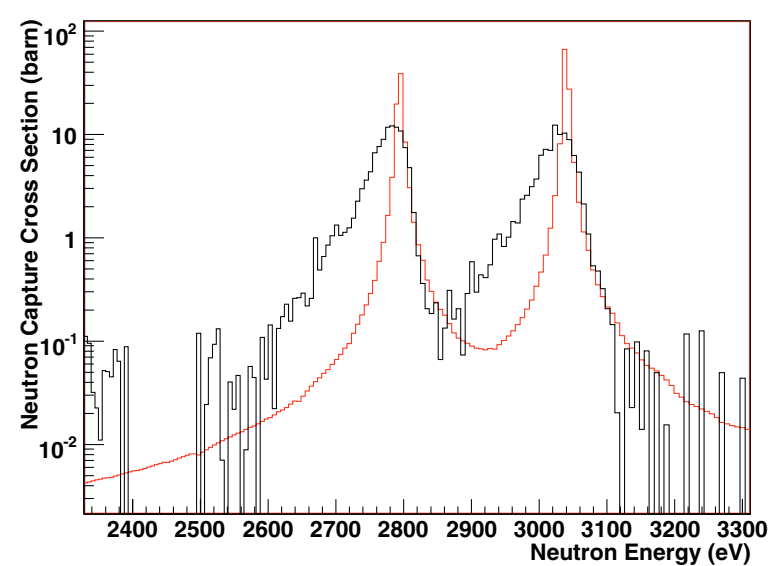

Fig. 10. Neutron capture cross section for ${ }^{205} \mathrm{Tl}$ derived from DANCE (black) (multiplicity cut from 2 to 3 and total-energy cut from 5 to $6.5 \mathrm{meV}$ ) and a comparison with evaluated data (red) [13] for the biggest resonance doublet in the ${ }^{205} \mathrm{Tl}(\mathrm{n}, \gamma)$ cross section (see fig. 9).

Figure 10 shows the conversion of the data to cross section in the neutron energy region around the resonance doublet of the ${ }^{205} \mathrm{Tl}(\mathrm{n}, \gamma)$ reaction. The plot illustrates the improved accuracy of the DANCE data compared to the data reported so far (see fig. 9). As for ${ }^{203} \mathrm{Tl}(\mathrm{n}, \gamma)$, the final data will be derived from the smaller sample 4 for the biggest resonances and from the bigger sample 3 elsewhere.

\section{Conclusion}

Neutron capture data on enriched samples of the stable isotopes of thallium have been taken with the DANCE array at the Los Alamos National Laboratory. The goal of the experiment was twofold. Firstly, we wanted to improve the available experimental data in the astrophysically interesting energy region between $100 \mathrm{eV}$ and $200 \mathrm{keV}$. Secondly, we wanted to prepare for the neutron capture measurement on the important $s$-process branch point ${ }^{204} \mathrm{Tl}$.

The preliminary data presented in this article suggest that both goals can be achieved as soon as the final data analysis has been performed.

This work has benefited from the use of the Los Alamos Neutron Science Center at the Los Alamos National Laboratory. This facility is funded by the US Department of Energy and operated by Los Alamos National Security, LLC under contract DE-AC5206NA25396. Prepared for the US Department of Energy Office of Nuclear Energy Under DOE Idaho Operations Office Contract DEAC07-05ID14517.

\section{References}

1. Z.Y. Bao, H. Beer, F. Käppeler, F. Voss, K. Wisshak, T. Rauscher, At. Data Nucl. Data Tables 76, 70 (2000).

2. K. Takahashi, K. Yokoi, At. Data Nucl. Data Tables 36, 375 (1987).

3. K. Yokoi, K. Takahashi, M. Arnould, A\&A 145, 339 (1985).

4. U. Ratzel, C. Arlandini, F. Käppeler, A. Couture, M. Wiescher, R. Reifarth, R. Gallino, A. Mengoni, C. Travaglio, Phys. Rev. C 70, 065803 (2004).

5. C. Arlandini, F. Käppeler, K. Wisshak, R. Gallino, M. Lugaro, M. Busso, O. Straniero, ApJ 525, 886 (1999).

6. P.W. Lisowski, C.D. Bowman, G.J. Russell, S.A. Wender, Nucl. Sci. Eng. 106, 208 (1990).

7. M. Heil, R. Reifarth, M.M. Fowler, R.C. Haight, F. Käppeler, R.S. Rundberg, E.H. Seabury, J.L. Ullmann, J.B. Wilhelmy, K. Wisshak et al., Nucl. Instrum. Meth. A 459, 229 (2001).

8. D. Habs, F.S. Stephens, R.M. Diamond, Tech. Rep., Report LBL8945, Lawrence Berkeley Laboratory (1979).

9. R. Reifarth, T.A. Bredeweg, A. Alpizar-Vicente, J.C. Browne, E.I. Esch, U. Greife, R.C. Haight, R. Hatarik, A. Kronenberg, J.M. O'Donnell et al., Nucl. Instrum. Meth. A 531, 528 (2004).

10. J.L. Ullmann, U. Agvaanluvsan, A. Alpizar-Vicente, E. Bond, T.A. Bredeweg, E.I. Esch, C.M. Folden, U. Greife, R. Hatarik, R.C. Haight et al., AIP Conf. Proc. 769, 918 (2005).

11. R.L. Macklin, R.R. Winters, ApJ 208, 812 (1976).

12. V.A. Konks, F.L. Shapiro, Soviet Physics JETP-USSR 20, 531 (1965).

13. NEA/EFF working group, Tech. Report JEFF Report 19, OECD Nuclear Energy Agency, www.nea.fr/html/dbdata/nds_jefreports/jefreport-19/jefreport19.pdf (2005).

14. Y.Y. Stavisskii, V.A. Tolstikov, Reactor Science and Technology (J. Nucl. Energy Parts A and B) 16, 496 (1962). 